\title{
Ultrafast laser inscribed 3D integrated photonics
}

\author{
S. Gross ${ }^{a}$, A. Arriola ${ }^{a}$, G. Palmer ${ }^{a, b}$, N. Jovanovic ${ }^{a, c}$, I. Spaleniak ${ }^{a}$, T. D. Meany ${ }^{a}$, Y. Duan ${ }^{a}$, \\ Q. Liu ${ }^{a}$, P. Dekker ${ }^{a}$, D. G. Lancaster ${ }^{d}$, H. Ebendorff-Heidepriem ${ }^{d}$, P. G. Tuthille ${ }^{e}$, B. Norris ${ }^{e}$, \\ A. Fuerbach ${ }^{a}$, M. Ireland ${ }^{a}$, M. J. Steel ${ }^{a}$ and M. J. Withford ${ }^{a}$ \\ ${ }^{a}$ Centre for Ultrahigh-bandwidth Devices for Optical Systems (CUDOS) and MQ Photonics \\ Research Centre, Department of Physics and Astronomy, Macquarie University, New South \\ Wales, 2109, Australia; \\ ${ }^{b}$ European XFEL GmbH, Albert-Einstein-Ring 19, 22761 Hamburg, Germany; \\ ${ }^{c}$ Subaru Telescope - National Astronomical Observatory of Japan (NAOJ), Hawaii, 96720, \\ USA; \\ ${ }^{d}$ IPAS and School of Chemistry \& Physics, University of Adelaide, South Australia, 5005, \\ Australia; \\ ${ }^{e}$ Sydney Institute for Astronomy (SIfA), Institute for Photonics and Optical Science (IPOS), \\ School of Physics, University of Sydney, New South Wales 2006, Australia
}

\begin{abstract}
Since the discovery, that a tightly focused femtosecond laser beam can induce a highly localized and permanent refractive index change in a wide range of dielectrics, ultrafast laser inscription has found applications in many fields due to its unique 3D and rapid prototyping capabilities. These ultrafast laser inscribed waveguide devices are compact and lightweight as well as inherently robust since the waveguides are embedded within the bulk material. In this presentation we will review our current understanding of ultrafast laser - glass lattice interactions and its application to the fabrication of inherently stable, compact waveguide lasers and astronomical 3D integrated photonic circuits.
\end{abstract}

Keywords: Ultrafast laser inscription, Laser materials processing, Burried waveguides, Astrophotonics, Waveguide lasers, Mid-infrared

\section{INTRODUCTION}

It was the progress in short pulse laser technology starting in the late 80s which enabled many new exciting scientific areas. The unique properties of ultrashort laser pulses led to the direct observation of chemical processes on a unprecedent time scale for the first time. ${ }^{1}$ The spectral characteristics of mode-locked lasers enabled the development of optical frequency combs ${ }^{2}$ linking radio frequency with the optical frequency domain and therefore facilitating the development of table-top optical atomic clocks for precision metrology. ${ }^{3}$ A femtosecond pulse can provide very high peak intensities and therefore enabling highly nonlinear processes. Furthermore, the energy can be deposited within timescales shorter than thermal processes. ${ }^{4}$ This allows for machining of materials, which are naturally transparent on a sub micrometer scale without any thermal damage.

These unique properties resulted in a wide range of research activities into femtosecond laser micromachining. Glezer et. al found that femtosecond pulses can lead to a explosive expansion when focused into fused silica, creating $200 \mathrm{~nm}$ sized voids within the bulk material. ${ }^{5}$ However, it was Davis et. al, who discovered that femtosecond pulses of lower energy can induce a highly localized and smooth refractive index change within bulk glass. ${ }^{6}$ They used $120 \mathrm{fs}, 810 \mathrm{~nm}$ pulses from a $200 \mathrm{kHz}$ titanium sapphire amplifier, which were focused into various types of glass, while the samples were moved by translation stages to inscribe lines of positive refractive index change, which acted as optical waveguides. This landmark paper led to a whole new research area of femtosecond laser direct-write photonics.

Further author information: (Send correspondence to S. Gross)

E-mail: simon.gross@mq.edu.edu, Telephone: +61 298505021

Nanophotonics and Macrophotonics for Space Environments VII, edited by

Edward W. Taylor, David A. Cardimona, Proc. of SPIE Vol. 8876, 887604

(C) 2013 SPIE $\cdot$ CCC code: $0277-786 X / 13 / \$ 18 \cdot$ doi: $10.1117 / 12.2025535$

Proc. of SPIE Vol. $8876887604-1$ 


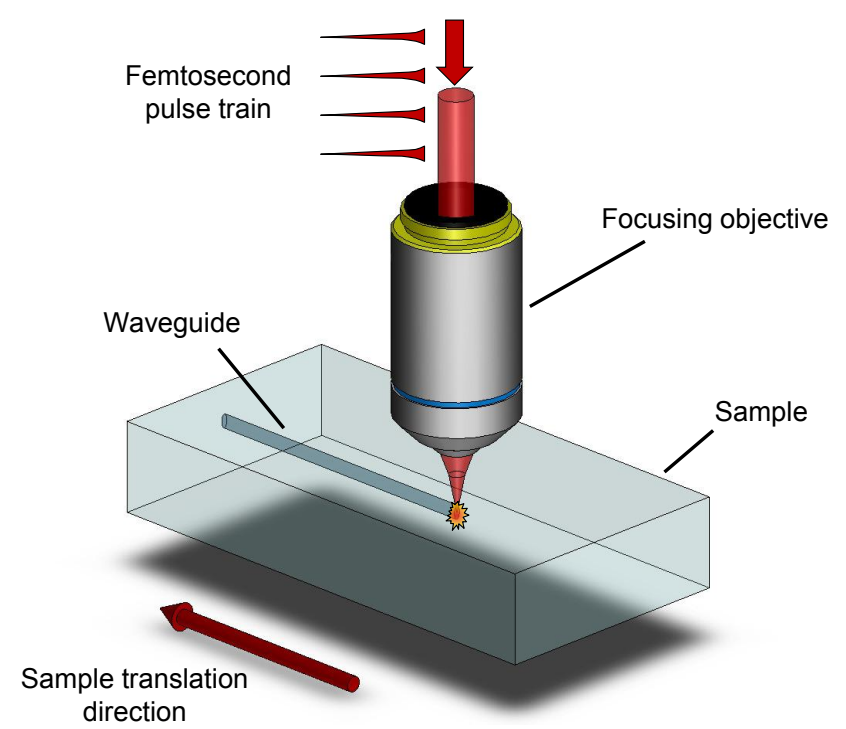

Figure 1. Ultrafast laser inscription setup: A femtosecond laser is tightly focused into the bulk of the sample, nonlinear breakdown occurs, which causes a localized material modification. By translating the sample with respect to the focal spot, arbitrary 3 dimensional structures can be inscribed.

The nonlinear absorption processes involved can virtually be triggered in any material, resulting in a highly localized modification at the position of the focal spot. The morphology of the modification strongly depends on the inscription parameters and the material itself. Nevertheless, waveguides have been inscribed in a wide range of transparent dielectrics, starting from common glasses, like fused silica, ${ }^{7}$ boro- and aluminosilicates, ${ }^{8,9}$ soda-lime,${ }^{10}$ to more exotic glasses, like phosphates, ${ }^{11}$ phospho-tellurite,${ }^{12}$ tellurite,${ }^{13}$ fluorogermanate ${ }^{14}$ various types of chalcogenides, ${ }^{15-17}$ fluorozirconate ${ }^{18}$ and bismuth borate, ${ }^{19}$ polymers, like PMMA ${ }^{20}$ and CYTOP ${ }^{21}$ up to crystals, like lithium niobate, ${ }^{22}$ silicon, ${ }^{23} \mathrm{YAG}^{24}{ }^{24} \mathrm{YVO}_{4},{ }^{25}$ tungstates $,{ }^{26,27} \mathrm{KTP},{ }^{28} \mathrm{BiB}_{3} \mathrm{O}_{6}{ }^{29}$ and sapphire. ${ }^{30}$ Together with its rapid prototyping and $3 \mathrm{D}$ capabilities it allowed for the realisation of a huge variety of devices, like the already mentioned simple waveguides, ${ }^{6}$ couplers, ${ }^{31,32}$ Fresnel lenses,${ }^{33}$ waveguide Bragg gratings, ${ }^{34}$ waveguide amplifier ${ }^{35}$ monolithic waveguide lasers, ${ }^{36}$ photonic quantum circuits, ${ }^{37}$ astrophotonic circuits ${ }^{38}$ and sensors. ${ }^{10,39}$

\section{FEMTOSECOND PULSE INDUCED STRUCTURAL MODIFICATIONS}

The principle ultrafast laser inscription setup is illustrated in Fig. 1. The output of a femtosecond laser is tightly focused by a lens into the bulk material. By placing the sample on translation stages and moving it with respect to the focal spot, arbitrary 3-dimensional structures can be inscribed. The structural modifications within the transparent dielectric induced by the tightly focused laser beam depend on parameters such as the laser wavelength, repetition rate, pulse duration, polarization, focusing condition, translation speed and of course the material itself. Hence, there is no universal recipe for waveguide inscription, instead the parameters for inscribing high quality waveguides have to be determined empirically for every material.

Key to the optimization of the inscription parameters is a deeper understanding of the morphology of the laser induced structural modifications. In the case of optical glasses, this will allow for the adaption of the glass composition in order to trigger a specific response to the exposure of sub-bandgap femtosecond radiation. This response can be a specific magnitude or sign of refractive index change. The femtosecond laser induced structural changes have been investigated for a variety of materials using techniques such as fluorescence and Raman spectroscopy. For instance in fused silica the increase of refractive after irradiation with $\mathrm{kHz}$ repetition rate femtosecond laser pulses was attributed to an increase in 3-membered silicon oxygen rings. ${ }^{40}$ In phosphate glass an increased number of $Q^{1}$ phosphorous tetrahedra (one bridging oxygen) was found after femtosecond pulse irradiation. ${ }^{41}$ This leads to an increased polarizability of the glass network and thus an increase in refractive index. The repetition rate has a significant influence on the morphology of index change. For instance when irradiating 
BK7, a multi-component silicate glass, with high repetition rate pulses a densification of the glass network and therefore an increase of refractive index was found whereas using low repetition rate pulses non-bridging oxygen hole centers are created that are responsible for the increased index. ${ }^{42}$ The difference in structural change can be related to the thermal condition. With low repetition rate exposure (athermal regime), the glass is quenched on a timescale in the order of the thermal diffusion time $(\approx 1 \mu \mathrm{s})$ and the glass cools down to ambient temperature before the next pulse strikes, as illustrated in Fig. 2. In contrast, high repetition rate irradiation results in an accumulation of heat and strong heat diffusion up to melting of the glass. ${ }^{9}$ As the sample is translated through the focus at speeds typical in the order of $\mathrm{mm} / \mathrm{s}$ the glass cools and resolidifies on a millisecond timescale. The localized melting can result in migration of elements driven by thermodiffusion. ${ }^{43}$ This generally gives rise to a highly temperature stable $\left(>600{ }^{\circ} \mathrm{C}\right)$ refractive index change. ${ }^{44}$ In contrast, athermal written structures anneal out at temperatures of $400{ }^{\circ} \mathrm{C} .{ }^{45}$

Besides the laser's repetition rate, also the polarization state of the inscription laser can have a significant influ-

\section{Athermal regime}

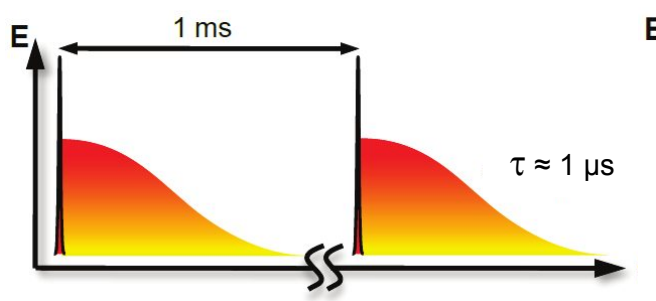

Thermal regime

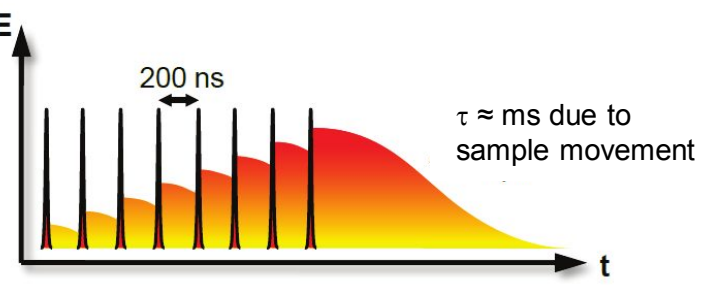

Figure 2. Difference between the athermal (low laser repetition rate) and thermal writing regime (high laser repetition rate).

ence on the morphology of the refractive index change. The laser polarization not only affects the photoionization process $^{46}$ but can also trigger the creation of self-aligned nanogratings with a period below the wavelength of the inscription laser. ${ }^{47}$ The planes of these nanogratings are aligned perpendicular to the electric field vector for linear polarization and result in form birefringence. This effect can be utilized to create retardation plates, ${ }^{48}$ optical storage devices, ${ }^{49}$ or integrated polarization beam splitters. ${ }^{50}$

The multitude of parameters that influence the final device requires a careful choice of writing parameters and substrate material for each application individually. For instance utilizing a high repetition rate laser and a suitable glass is advantageous for fabricating complex devices, since the thermal regime offers vastly faster fabrication speeds than athermal regime. Therefore the fabrication process is less influenced by changing environmental conditions, for instance room temperature or drift in laser output power. However, the strong heat diffusion in the thermal regime makes the fabrication of sub-micron features like waveguide Bragg gratings challenging. Hence, the athermal fabrication regime is preferred in that case.

The following section will outline some 3D applications for ultrafast laser inscription. Since the presented devices are quite complex, all waveguides were fabricated in the thermal regime.

\section{APPLICATIONS}

\subsection{Astrophotonics}

Astrophotonics is one of the main driving factors for 3D integrated photonics. ${ }^{51}$ The unique 3D capabilities of ultrafast laser inscription make it very attractive for photonic device fabrication for applications such as aperture masking interferometry ${ }^{52}$ or multimode light to single-mode light converters for atmospheric $\mathrm{OH}$ emission filtering ${ }^{53}$ and integrated photonic spectrographs. ${ }^{54}$

\subsubsection{Integrated pupil remapper}

High contrast imaging such as aperture masking interferometry ${ }^{52,55}$ allows for the detection of faint extra-solar planets nearby their parent star. In contrast to indirect detection techniques like the well established transit and Doppler technique, it enables the study of planets in any orbit/period. However the shortcomings of aperture masking interferometry are a limited throughput and Fourier coverage as well as leakage of residual atmospheric 


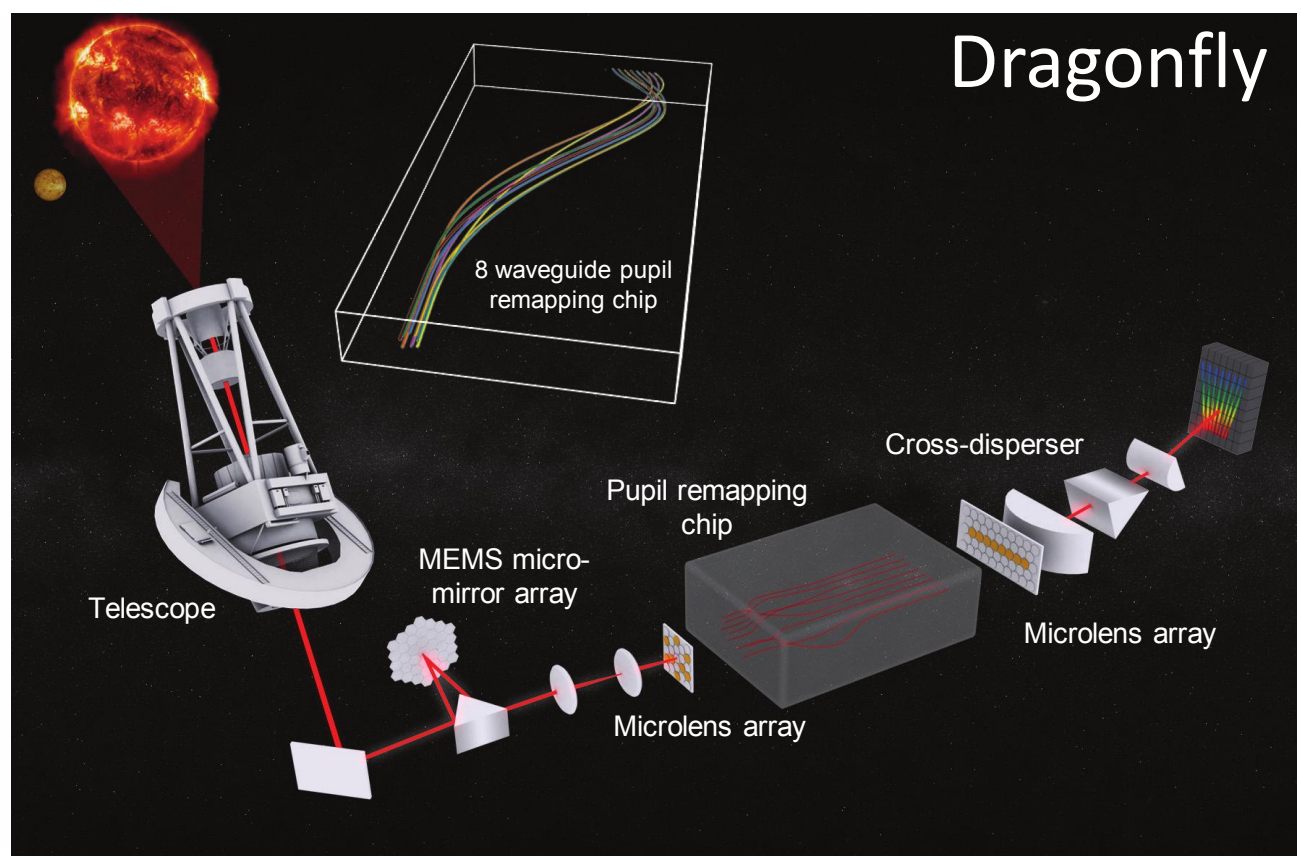

Figure 3. Artist's rendering of the Dragonfly instrument: An integrated pupil remapper using a 3D photonic chip. The ultrafast laser inscribed photonic chip remaps 8 input waveguides into an linear array at output while maintaining identical optical pathlength across all waveguides.

noise due to phase corrugations across each sub-aperture. These limitations can be overcome by using photonic technologies such as single-mode waveguides to remap a 2D pupil into a linear waveguide array (an operation referred as pupil remapping). In principal, this allows for remapping the entire telescope pupil which not only increases the Fourier coverage but also the throughput compared to a sparsely sampled aperture mask. By cross-dispersing the light emerging from the linear 1 dimensional output waveguide array, spatial and spectral information can be simultaneously extracted from the created fringe pattern on the detector which is not possible with an aperture mask due to its 2 dimensional nature. Since the single-mode waveguides act as spatial filters, the noise induced by the atmosphere on the fringe visibility is reduced. The principal setup of such an instrument is shown in Fig. $3 .^{55}$ At its heart is the 3D integrated pupil remapping photonic chip, which remaps eight waveguides into a linear slit while maintaining path length matching and limited cross coupling between the waveguides. The most recent version of these pupil remapping chips have raw throughputs of $>70 \%$ at $1.55 \mu \mathrm{m}$ and $<10^{-5}$ cross-coupling. The waveguides are inscribed into a commercial boro-aluminosilicate glass which responds with a positive refractive index change $\left(\Delta n>5 \times 10^{-3}\right)$ upon high repetition rate pulse exposure.

\subsubsection{Photonic lanterns}

The next generation of ground-based astronomical telescopes with primary mirror diameters in excess of $20 \mathrm{~m}$ will require increasingly larger instruments, such as spectrographs for instance where their size scales proportional to the telescope aperture. However the size of these instruments is the driving factor for the costs, which scale with the square of the telescope aperture. ${ }^{56}$ A potential solution is the use of photonic instruments such as integrated photonic spectrographs (arrayed waveguide gratings). ${ }^{54}$ However these components are single-mode (diffraction limited) devices whereas light from the telescope is multimode (seeing limited) if no adaptive optics are fitted. Therefore in order to use cost effective single-mode photonic components a converter from multimode to single-mode light is required. Such a device is a photonic lantern, a low loss adiabatic tapered transition which converts the light from a multimode fiber into several single-mode fibers. ${ }^{57}$ The first photonic lanterns were fiber based, but more recently ultrafast laser inscribed lanterns were demonstrated. ${ }^{38}$ Using ultrafast laser inscription not only results in a highly robust component since it is embedded within a block of glass, but also provides greater geometric flexibility ${ }^{58}$ than the fiber counterparts and can easily be scaled to a large number of 


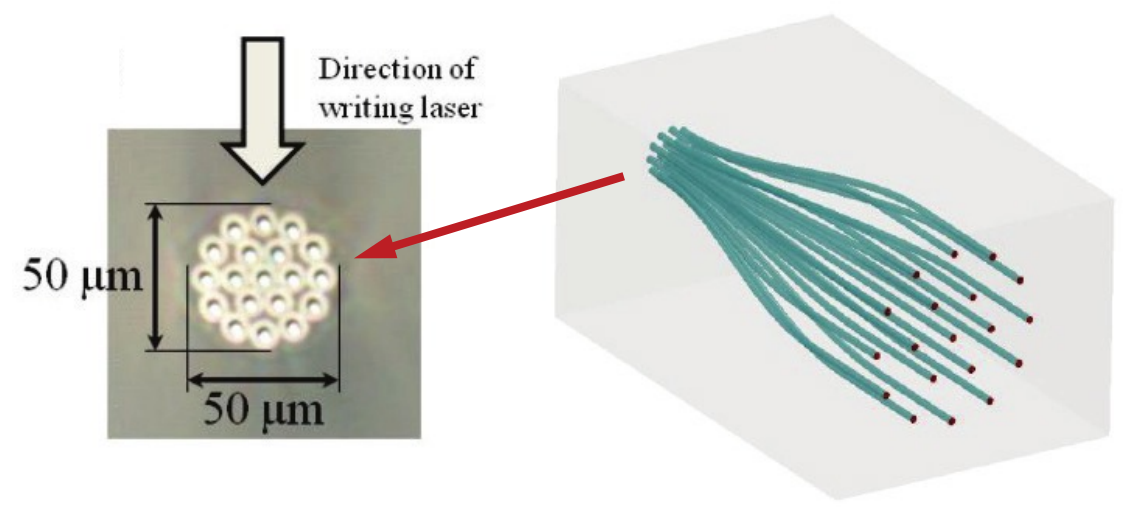

Figure 4. Laser inscribed photonic lantern with 19 single-mode waveguides, which are brought close together at the input to form a multimode waveguide.

waveguides (>100) since no stacking of single-mode fibers is involved. The laser inscribed photonic lanterns are composed of 19 single-mode waveguides, brought close together in a circular arrangement at the input to form a multimode waveguide ${ }^{59}$ (see Fig. 4). These devices have been carefully optimized for throughput to provide raw transmission values of $75 \%$.

\subsubsection{Low loss mid-IR waveguides}

The mid-infrared spectral region from 3.5 to $4.2 \mu \mathrm{m}$ (astronomical L'-band) is ideal for hunting for young extrasolar planets. This is because the young planet is still hot from the formation process and thus has its peak of the blackbody radiation curve in the mid-infrared. Hence the difference in brightness between the parent star and its companion decreases, since the star is less bright in that spectral region. Even though the brightness difference becomes even smaller further in the mid-infrared, the thermal background at longer wavelength increases the
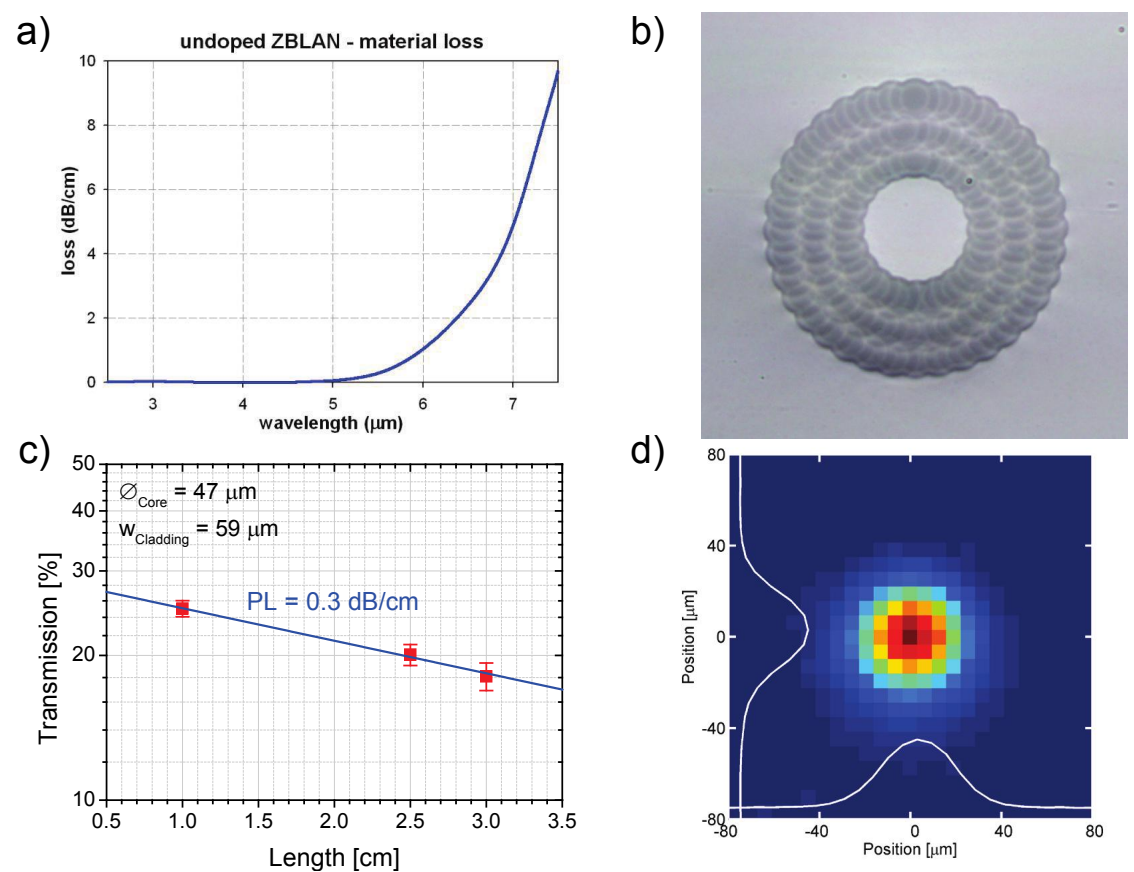

d)

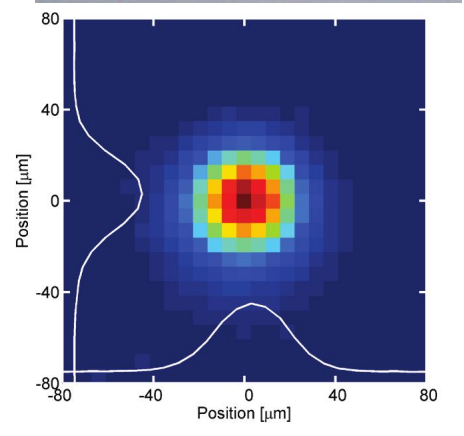

Figure 5. Laser written mid-infrared waveguides in ZBLAN glass. (a) ZBLAN material loss. (b) End on optical microscope images of a depressed cladding waveguide. (c) Waveguide propagation losses and near-field profile (d) at $4 \mu \mathrm{m}$. 
noise and makes ground-based observation more challenging. Finally, there is an atmospheric transmission window in the 3-4 $\mu \mathrm{m}$ region which allows for ground-based observation.

In order to transfer the previously mentioned devices into the mid-infrared region a different host material is required because silica based glasses are not transparent beyond $3 \mu \mathrm{m}$ wavelength. An excellent candidate is fluoride glass, ${ }^{60}$ in particular ZBLAN, a fluorozirconate glass with an optical transmission window from $200 \mathrm{~nm}$ all the way up to $4.5 \mu \mathrm{m}$, as illustrated in Fig. 5(a). The high infrared transparency results from the low phonon energy of the glass. Besides the high transparency, the glass features a low refractive index of 1.5 in the visible spectral region and also has a low optical nonlinearity. The latter is of importance when focusing high peak power femtosecond pulses into to glass for waveguide writing, where nonlinear propagation effects can aberrate the beam resulting in poor quality waveguides.

When irradiating ZBLAN with high repetition femtosecond pulses a decrease in refractive index is observed. ${ }^{61,62}$ Therefore in order to create a guiding structure a depressed cladding has to be inscribed, as shown in Fig. 5(b). The geometrical flexibility of the depressed cladding approach enables the creation of perfectly circular guiding structures with a variable size to accommodate for the longer operation wavelength. Since the core, which the guided light is confined to, stays unmodified the glass bulk optical properties are preserved. Therefore a low propagation loss of $0.3 \mathrm{~dB} / \mathrm{cm}$ is achievable (Fig. $5(\mathrm{c})$ ) at $4 \mu \mathrm{m}$. Figure $5(\mathrm{~d}$ ) shows the corresponding single-mode near-field profile, which is of perfect circular symmetry that makes it ideal for efficiently injecting light from an astronomical telescope.

\subsection{High efficiency mid-IR waveguide lasers}

Besides astronomy, the mid-infrared spectral region is of great interest for spectroscopy, remote sensing and for medical applications, since it coincides with the characteristic absorption lines of the vast majority of gaseous molecules. This enables the specific detection of a molecular species via its spectroscopic fingerprint. Furthermore these absorption features can also be targeted for energy deposition and material ablation. For instance this is the case in the field of medicine where the water absorption bands around $2 \mu \mathrm{m}$ and $3 \mu \mathrm{m}$ are utilized for precise and minimal invasive procedures in dentistry, neurosurgery, dermatology and ophthalmology. All of these applications benefit from the availability of compact, efficient, robust and cost effective laser sources such as waveguide lasers.

\section{a)}

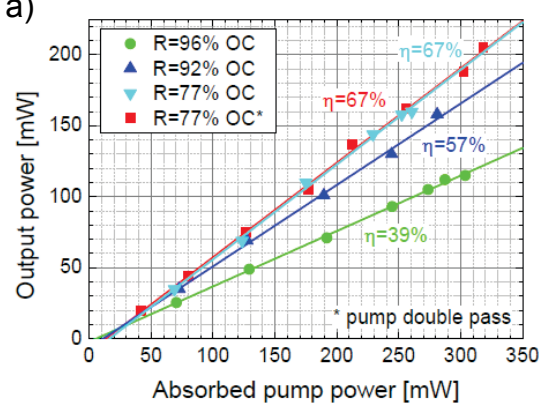

c)

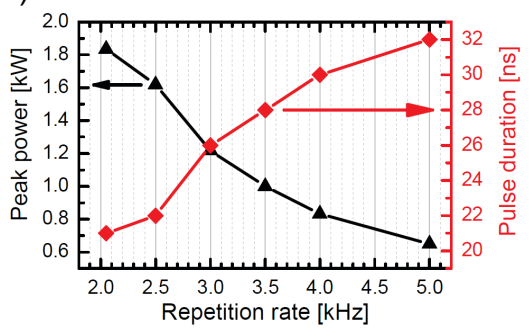

b)

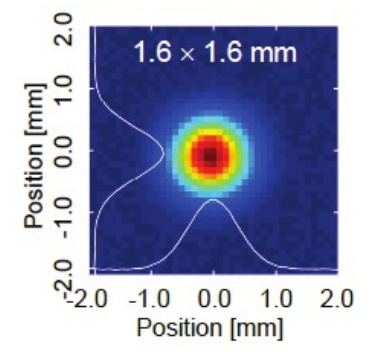

d)

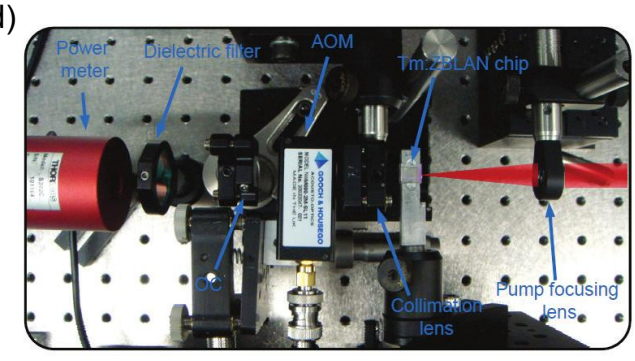

Figure 6. (a) Continuous wave performance of a thulium doped ZBLAN waveguide laser emitting at $1.9 \mu \mathrm{m}$. (b) Corresponding collimated output beam profile. (c) Q-switched performance of an identical thulium doped ZBLAN waveguide chip placed in an external cavity to accommodate an acousto-optic modulator. (d) Photograph of the external cavity. 
Using ultrafast laser inscription for the fabrication of waveguide laser is attractive because it is not only compatible with a large range of materials but it also enables the direct incorporation of Bragg gratings to create a monolithic device. ${ }^{36}$ In particular ZBLAN is very good candidate as host material. As aforementioned, ZBLAN glass possess a low phonon energy, which limits non-radiative losses and therefore enables high laser efficiency in the mid-infrared. Furthermore the glass matrix can accommodate large quantities of rare-earth dopants, which is ideal for short high gain waveguide chips. Doping ZBLAN with rare-earth ions like ytterbium, thulium, holmium and erbium enables to cover a large portion of the wavelength region from 1 to $3 \mu \mathrm{m} .{ }^{63}$ We have successfully applied the depressed cladding waveguide architecture to create fingernail-sized waveguide laser emitting at 1 , 1.9, 2.1 and $2.9 \mu \mathrm{m} .{ }^{64-68}$ In particular, lasers emitting at $1.9 \mu \mathrm{m}$ based on thulium doped ZBLAN can be very efficient, reaching slope efficiencies of $67 \%$ in continuous wave operation at $>200 \mathrm{~mW}$ average output power (Fig. 6(a)). Due to the depressed cladding waveguides the output beam is circular and diffraction limited (Fig. 6(b)). Furthermore they enable large mode areas while maintaining the beam quality, which lead to $1.8 \mathrm{~kW}$ peak power in q-switched operation from a waveguide chip laser, as illustrated in Fig. 6(c). ${ }^{67}$ The pulse duration was as short as $21 \mathrm{~ns}$ limited by the acousto-optic modulator placed inside the $9 \mathrm{~cm}$ long external cavity (Fig. 6(d)).

\section{CONCLUSION}

Ultrafast laser inscription is a powerful tool for the fabrication of photonic circuitry. It enables the creation of active and passive 3-dimensional photonic devices with operation wavelengths ranging from the visible all the way up to the mid-infrared spectral region. With the appropriate choice of inscription parameters high quality, low loss waveguides can be fabricated in a wide variety of materials. These waveguide devices are compact and inherently robust to environmental influences such as mechanical vibration and temperature because the optical waveguides are embedded within the bulk material. The unique capabilities of ultrafast laser inscription have drawn a lot of attention from a variety of fields like astrophotonics, quantum optics and microfluidics, but might also be beneficial for potential future applications such as space photonics.

\section{ACKNOWLEDGMENTS}

This research was supported by the Australian Research Council Centre of Excellence for Ultrahigh bandwidth Devices for Optical Systems (project number CE110001018) and was performed in part at the OptoFab node of the Australian National Fabrication Facility utilizing Commonwealth and NSW State Government funding.

\section{REFERENCES}

[1] Zewail, A. H., "Femtochemistry: Atomic-Scale Dynamics of the Chemical Bond," The Journal of Physical Chemistry A 104, 5660-5694 (June 2000).

[2] Udem, T., Holzwarth, R., and Hänsch, T. W., "Optical frequency metrology," Nature 416, $233-7$ (Mar. 2002).

[3] Newbury, N. R., "Searching for applications with a fine-tooth comb," Nature Photonics 5, 186-188 (Apr. 2011).

[4] Gattass, R. R. and Mazur, E., "Femtosecond laser micromachining in transparent materials," Nature Photonics 2, 219-225 (Apr. 2008).

[5] Glezer, E. N., Milosavljevic, M., Huang, L., Finlay, R. J., Her, T.-H., Callan, J. P., and Mazur, E., "Threedimensional optical storage inside transparent materials," Optics Letters 21, 2023 (Dec. 1996).

[6] Davis, K. M., Miura, K., Sugimoto, N., and Hirao, K., "Writing waveguides in glass with a femtosecond laser.," Optics Letters 21, 1729-31 (Nov. 1996).

[7] Ams, M., Marshall, G. D., and Withford, M. J., "Study of the influence of femtosecond laser polarisation on direct writing of waveguides.," Optics Express 14, 13158-63 (Dec. 2006).

[8] Eaton, S. M., Ng, M. L., Bonse, J., Mermillod-Blondin, A., Zhang, H., Rosenfeld, A., and Herman, P. R., "Low-loss waveguides fabricated in BK7 glass by high repetition rate femtosecond fiber laser.," Applied Optics 47, 2098-102 (Apr. 2008). 
[9] Eaton, S. M., Zhang, H., Ng, M. L., Li, J., Chen, W.-J., Ho, S., and Herman, P. R., "Transition from thermal diffusion to heat accumulation in high repetition rate femtosecond laser writing of buried optical waveguides.," Optics Express 16, 9443-58 (June 2008).

[10] Kamata, M., Obara, M., Gattass, R. R., Cerami, L. R., and Mazur, E., "Optical vibration sensor fabricated by femtosecond laser micromachining," Applied Physics Letters 87(5), 051106 (2005).

[11] Cerullo, G., Osellame, R., Taccheo, S., Marangoni, M., Polli, D., Ramponi, R., Laporta, P., and De Silvestri, S., "Femtosecond micromachining of symmetric waveguides at 1.5 microm by astigmatic beam focusing.," Optics Letters 27, 1938-40 (Nov. 2002).

[12] Fernandez, T. T., Della Valle, G., Osellame, R., Jose, G., Chiodo, N., Jha, A., and Laporta, P., "Active waveguides written by femtosecond laser irradiation in an erbium-doped phospho-tellurite glass.," Optics Express 16, 15198-205 (Sept. 2008).

[13] Nandi, P., Jose, G., Jayakrishnan, C., Debbarma, S., Chalapathi, K., Alti, K., Dharmadhikari, a. K., Dharmadhikari, J. a., and Mathur, D., "Femtosecond laser written channel waveguides in tellurite glass.," Optics Express 14, 12145-50 (Dec. 2006).

[14] Fusari, F., Thomson, R. R., Jose, G., Bain, F. M., Lagatsky, A. A., Psaila, N. D., Kar, A. K., Jha, A., Sibbett, W., and Brown, C. T., "Lasing action at around $1.9 \mu \mathrm{m}$ from an ultrafast laser inscribed Tm-doped glass waveguide," Optics Letters 36, 1566 (Apr. 2011).

[15] Zoubir, A., Richardson, M., Rivero, C., Schulte, A., Lopez, C., Richardson, K., Hô, N., and Vallée, R., "Direct femtosecond laser writing of waveguides in As2S3 thin films.," Optics Letters 29, 748-50 (Apr. 2004).

[16] Psaila, N. D., Thomson, R. R., Bookey, H. T., Shen, S., Chiodo, N., Osellame, R., Cerullo, G., Jha, A., and Kar, A. K., "Supercontinuum generation in an ultrafast laser inscribed chalcogenide glass waveguide.," Optics Express 15, 15776-81 (Nov. 2007).

[17] Hughes, M. A., Yang, W., and Hewak, D. W., "Spectral broadening in femtosecond laser written waveguides in chalcogenide glass," Journal of the Optical Society of America B 26, 1370 (June 2009).

[18] Miura, K., Qiu, J., Mitsuyu, T., and Hirao, K., "Preparation and optical properties of fluoride glass waveguides induced by laser pulses," Journal of Non-Crystalline Solids 256-257, 212-219 (Oct. 1999).

[19] Yang, W., Corbari, C., Kazansky, P. G., Sakaguchi, K., and Carvalho, I. C. S., "Low loss photonic components in high index bismuth borate glass by femtosecond laser direct writing.," Optics Express 16, 16215-26 (Sept. 2008).

[20] Watanabe, W., Sowa, S., Tamaki, T., Itoh, K., and Nishii, J., "Three-Dimensional Waveguides Fabricated in Poly(methyl methacrylate) by a Femtosecond Laser," Japanese Journal of Applied Physics 45, L765-L767 (July 2006).

[21] Hanada, Y., Sugioka, K., and Midorikawa, K., "UV waveguides light fabricated in fluoropolymer CYTOP by femtosecond laser direct writing.," Optics Express 18, 446-50 (Jan. 2010).

[22] Thomas, J., Heinrich, M., Zeil, P., Hilbert, V., Rademaker, K., Riedel, R., Ringleb, S., Dubs, C., Ruske, J.-P., Nolte, S., and Tünnermann, A., "Laser direct writing: Enabling monolithic and hybrid integrated solutions on the lithium niobate platform," Physica Status Solidi (a) 208, 276-283 (Feb. 2011).

[23] Nejadmalayeri, A. H., Herman, P. R., Burghoff, J., Will, M., Nolte, S., and Tünnermann, A., "Inscription of optical waveguides in crystalline silicon by mid-infrared femtosecond laser pulses.," Optics Letters $\mathbf{3 0}$, 964-6 (May 2005).

[24] Okhrimchuk, A. G., Shestakov, A. V., Khrushchev, I., and Mitchell, J., "Depressed cladding, buried waveguide laser formed in a YAG:Nd3+ crystal by femtosecond laser writing.," Optics Letters 30, 2248-50 (Sept. 2005).

[25] Silva, W. F., Jacinto, C., Benayas, A., Vazquez de Aldana, J. R., Torchia, G. A., Chen, F., Tan, Y., and Jaque, D., "Femtosecond-laser-written, stress-induced Nd:YVO4 waveguides preserving fluorescence and Raman gain," Optics Letters 35, 916 (Mar. 2010).

[26] Borca, C., Apostolopoulos, V., Gardillou, F., Limberger, H., Pollnau, M., and Salathe, R., "Buried channel waveguides in Yb-doped KY(WO4)2 crystals fabricated by femtosecond laser irradiation," Applied Surface Science 253, 8300-8303 (July 2007). 
[27] Eaton, S. M., Merchant, C. A., Iyer, R., Zilkie, A. J., Helmy, A. S., Aitchison, J. S., Herman, P. R., Kraemer, D., Miller, R. J. D., Hnatovsky, C., and Taylor, R. S., "Raman gain from waveguides inscribed in KGd(WO4)2 by high repetition rate femtosecond laser," Applied Physics Letters 92(8), 081105 (2008).

[28] Campbell, S., Thomson, R. R., Hand, D. P., Kar, A. K., Reid, D. T., Canalias, C., Pasiskevicius, V., and Laurell, F., "Frequency-doubling in femtosecond laser inscribed periodically-poled potassium titanyl phosphate waveguides.," Optics Express 15, 17146-50 (Dec. 2007).

[29] Beecher, S. J., Thomson, R. R., Reid, D. T., Psaila, N. D., Ebrahim-Zadeh, M., and Kar, A. K., "Strain field manipulation in ultrafast laser inscribed $\mathrm{BiB} 3 \mathrm{O} 6$ optical waveguides for nonlinear applications.," Optics Letters 36, 4548-50 (Dec. 2011).

[30] Apostolopoulos, V., Laversenne, L., Colomb, T., Depeursinge, C., Salathe, R. P., Pollnau, M., Osellame, R., Cerullo, G., and Laporta, P., "Femtosecond-irradiation-induced refractive-index changes and channel waveguiding in bulk Ti3+:Sapphire," Applied Physics Letters 85(7), 1122 (2004).

[31] Homoelle, D., Wielandy, S., Gaeta, a. L., Borrelli, N. F., and Smith, C., "Infrared photosensitivity in silica glasses exposed to femtosecond laser pulses.," Optics Letters 24, 1311-3 (Sept. 1999).

[32] Liu, J., Zhang, Z., Chang, S., Flueraru, C., and Grover, C. P., "Directly writing of 1-to-N optical waveguide power splitters in fused silica glass using a femtosecond laser," Optics Communications 253, 315-319 (Sept. 2005).

[33] Yamada, K., Watanabe, W., Li, Y., Itoh, K., and Nishii, J., "Multilevel phase-type diffractive lenses in silica glass induced by filamentation of femtosecond laser pulses.," Optics Letters 29, 1846-8 (Aug. 2004).

[34] Marshall, G. D., Ams, M., and Withford, M. J., "Direct laser written waveguide-Bragg gratings in bulk fused silica.," Optics Letters 31, 2690-1 (Sept. 2006).

[35] Della Valle, G., Osellame, R., Chiodo, N., Taccheo, S., Cerullo, G., Laporta, P., Killi, A., Morgner, U., Lederer, M., and Kopf, D., "C-band waveguide amplifier produced by femtosecond laser writing.," Optics Express 13, 5976-82 (Aug. 2005).

[36] Marshall, G. D., Dekker, P., Ams, M., Piper, J. A., and Withford, M. J., "Directly written monolithic waveguide laser incorporating a distributed feedback waveguide-Bragg grating.," Optics Letters 33, 956-8 (May 2008).

[37] Marshall, G. D., Politi, A., Matthews, J. C. F., Dekker, P., Ams, M., Withford, M. J., and O'Brien, J. L., "Laser written waveguide photonic quantum circuits.," Optics Express 17, 12546-54 (July 2009).

[38] Thomson, R. R., Birks, T. A., Leon-Saval, S. G., Kar, A. K., and Bland-Hawthorn, J., "Ultrafast laser inscription of an integrated photonic lantern," Optics Express 19, 5698-705 (Mar. 2011).

[39] Vazquez, R. M., Osellame, R., Nolli, D., Dongre, C., van den Vlekkert, H., Ramponi, R., Pollnau, M., and Cerullo, G., "Integration of femtosecond laser written optical waveguides in a lab-on-chip.," Lab on a chip $\mathbf{9}$, 91-6 (Jan. 2009).

[40] Reichman, W. J., Krol, D. M., Shah, L., Yoshino, F., Arai, A., Eaton, S. M., and Herman, P. R., "A spectroscopic comparison of femtosecond-laser-modified fused silica using kilohertz and megahertz laser systems," Journal of Applied Physics 99(12), 123112 (2006).

[41] Little, D. J., Ams, M., Dekker, P., Marshall, G. D., and Withford, M. J., "Mechanism of femtosecond-laser induced refractive index change in phosphate glass under a low repetition-rate regime," Journal of Applied Physics 108(3), 033110 (2010).

[42] Little, D. J., Ams, M., Gross, S., Dekker, P., Miese, C. T., Fuerbach, A., and Withford, M. J., "Structural changes in BK7 glass upon exposure to femtosecond laser pulses," Journal of Raman Spectroscopy 42, 715-718 (Apr. 2011).

[43] Shimizu, M., Sakakura, M., Kanehira, S., Nishi, M., Shimotsuma, Y., Hirao, K., and Miura, K., "Formation mechanism of element distribution in glass under femtosecond laser irradiation.," Optics Letters 36, 2161-3 (June 2011).

[44] Arriola, A., Gross, S., Jovanovic, N., Charles, N., Tuthill, P. G., Olaizola, S. M., Fuerbach, A., and Withford, M. J., "Low bend loss waveguides enable compact, efficient 3D photonic chips," Optics Express 21, 2978 (Jan. 2013).

[45] Streltsov, A. M. and Borrelli, N. F., "Study of femtosecond-laser-written waveguides in glasses," Journal of the Optical Society of America B 19, 2496 (Oct. 2002). 
[46] Little, D. J., Ams, M., and Withford, M. J., "Influence of bandgap and polarization on photo-ionization: guidelines for ultrafast laser inscription [Invited]," Optical Materials Express 1, 670 (July 2011).

[47] Shimotsuma, Y., Kazansky, P., Qiu, J., and Hirao, K., "Self-Organized Nanogratings in Glass Irradiated by Ultrashort Light Pulses," Physical Review Letters 91, 1-4 (Dec. 2003).

[48] Ramirez, L. P. R., Heinrich, M., Richter, S., Dreisow, F., Keil, R., Korovin, A. V., Peschel, U., Nolte, S., and Tünnermann, A., "Tuning the structural properties of femtosecond-laser-induced nanogratings," Applied Physics A 100, 1-6 (Apr. 2010).

[49] Shimotsuma, Y., Sakakura, M., Kazansky, P. G., Beresna, M., Qiu, J., Qiu, J., Miura, K., and Hirao, K., "Ultrafast manipulation of self-assembled form birefringence in glass.," Advanced Materials 22, 4039-43 (Sept. 2010).

[50] Cheng, G., Mishchik, K., Mauclair, C., Audouard, E., and Stoian, R., "Ultrafast laser photoinscription of polarization sensitive devices in bulk silica glass," Optics Express 17, 9515 (May 2009).

[51] Bland-Hawthorn, J. and Kern, P., "Astrophotonics: a new era for astronomical instruments," Optics Express 17, 1880 (Jan. 2009).

[52] Baldwin, J. E., Haniff, C. A., Mackay, C. D., and Warner, P. J., "Closure phase in high-resolution optical imaging," Nature 320, 595-597 (Apr. 1986).

[53] Trinh, C. Q., Ellis, S. C., Bland-Hawthorn, J., Lawrence, J. S., Horton, A. J., Leon-Saval, S. G., Shortridge, K., Bryant, J., Case, S., Colless, M., Couch, W., Freeman, K., Löhmannsröben, H.-G., Gers, L., Glazebrook, K., Haynes, R., Lee, S., OByrne, J., Miziarski, S., Roth, M. M., Schmidt, B., Tinney, C. G., and Zheng, J., "Gnosis: the First Instrument To Use Fiber Bragg Gratings for Oh Suppression," The Astronomical Journal 145, 51 (Feb. 2013).

[54] Cvetojevic, N., Lawrence, J. S., Ellis, S. C., Bland-Hawthorn, J., Haynes, R., and Horton, A., "Characterization and on-sky demonstration of an integrated photonic spectrograph for astronomy.," Optics Express 17, 18643-50 (Oct. 2009).

[55] Jovanovic, N., Tuthill, P. G., Norris, B., Gross, S., Stewart, P., Charles, N., Lacour, S., Ams, M., Lawrence, J. S., Lehmann, a., Niel, C., Robertson, J. G., Marshall, G. D., Ireland, M., Fuerbach, a., and Withford, M. J., "Starlight demonstration of the Dragonfly instrument: an integrated photonic pupil-remapping interferometer for high-contrast imaging," Monthly Notices of the Royal Astronomical Society 427, 806-815 (Nov. 2012).

[56] Bland-Hawthorn, J. and Horton, A., "Instruments without optics: an integrated photonic spectrograph," in [Proceedings of SPIE], McLean, I. S. and Iye, M., eds., 62690N-62690N-14 (June 2006).

[57] Leon-Saval, S. G., Birks, T. A., Bland-Hawthorn, J., and Englund, M., "Multimode fiber devices with single-mode performance," Optics Letters 30(19), 2545 (2005).

[58] Fontaine, N. K., Ryf, R., Bland-Hawthorn, J., and Leon-Saval, S. G., "Geometric requirements for photonic lanterns in space division multiplexing," Optics Express 20, 27123 (Nov. 2012).

[59] Jovanovic, N., Spaleniak, I., Gross, S., Ireland, M., Lawrence, J. S., Miese, C., Fuerbach, A., and Withford, M. J., "Integrated photonic building blocks for next-generation astronomical instrumentation I: the multimode waveguide," Optics Express 20, 17029 (July 2012).

[60] Parker, J. M., "Fluoride Glasses," Annual Review of Materials Science 19, 21-41 (Aug. 1989).

[61] Gross, S., Ams, M., Palmer, G., Miese, C. T., Williams, R. J., Marshall, G. D., Fuerbach, A., Lancaster, D. G., Ebendorff-Heidepriem, H., and Withford, M. J., "Ultrafast Laser Inscription in Soft Glasses: A Comparative Study of Athermal and Thermal Processing Regimes for Guided Wave Optics," International Journal of Applied Glass Science 3, 332-348 (Dec. 2012).

[62] Gross, S., Lancaster, D. G., Ebendorff-Heidepriem, H., Monro, T. M., Fuerbach, A., and Withford, M. J., "Femtosecond laser induced structural changes in fluorozirconate glass," Optical Materials Express 3, 574 (Apr. 2013).

[63] Zhu, X. and Peyghambarian, N., "High power ZBLAN glass fiber lasers: review and prospect," Advances in OptoElectronics 2010, 1-23 (2010).

[64] Palmer, G., Gross, S., Fuerbach, A., Lancaster, D. G., and Withford, M. J., "High slope efficiency and high refractive index change in direct-written $\mathrm{Yb}$-doped waveguide lasers with depressed claddings," Optics Express 21, 17413 (July 2013). 
[65] Lancaster, D. G., Gross, S., Ebendorff-Heidepriem, H., Kuan, K., Monro, T. M., Ams, M., Fuerbach, A., and Withford, M. J., "Fifty percent internal slope efficiency femtosecond direct-written Tm3+:ZBLAN waveguide laser," Optics Letters 36, 1587 (Apr. 2011).

[66] Lancaster, D. G., Gross, S., Ebendorff-Heidepriem, H., Fuerbach, A., Withford, M. J., and Monro, T. M., "2.1 $\mu \mathrm{m}$ waveguide laser fabricated by femtosecond laser direct-writing in Ho3+, Tm3+:ZBLAN glass," Optics Letters 37, 996 (Mar. 2012).

[67] Lancaster, D. G., Gross, S., Fuerbach, A., Heidepriem, H. E., Monro, T. M., and Withford, M. J., "Versatile large-mode-area femtosecond laser-written Tm:ZBLAN glass chip lasers," Optics Express 20, 27503 (Nov. 2012).

[68] Lancaster, D. G., Gross, S., Ebendorff-Heidepriem, H., Withford, M. J., Monro, T. M., and Jackson, S. D., "Efficient $2.9 \mu \mathrm{m}$ fluorozirconate glass waveguide chip laser," Optics Letters 38, 2588 (July 2013). 